\title{
Scarring in molluscum contagiosum: comparison of physical expression and phenol ablation
}

\author{
Richard Weller, Chris J O’Callaghan, Ruth M MacSween, Marion I White
}

Department of Dermatology,

Royal Infirmary of Edinburgh, Edinburgh

EH3 9YW

Richard Weller specialist registrar

Ecology and

Epidemiology

Group, Department of Biological

Sciences, University of Warwick,

Coventry CV4 7AL

Chris J O'Callaghan epidemiologist

Department of Dermatology, Queen's Medical

Centre, Nottingham NG7 2UH

Ruth M MacSween specialist registrar

Department of

Dermatology,

Aberdeen Royal

Infirmary, Aberdeen

AB25 2ZN

Marion I White

consultant

dermatologist

Correspondence to: R M MacSween

Ruth_MacSween@ msn.com

BMJ 1999;319:1540

\begin{abstract}
Molluscum contagiosum is a benign viral skin disease occurring worldwide that commonly affects children. Spread may occur by autoinoculation or direct contact or via fomites. ${ }^{1}$ In immunocompetent individuals each lesion may last 6-8 weeks. With continuous autoinoculation, however, new lesions appear over time, such that the mean duration is about 8 months, ${ }^{2}$ with reports of infection lasting up to five years. ${ }^{3}$ Although resolution is ultimately spontaneous, scarring may occur, particularly if the lesions are secondarily infected. Thus there may be justification for active intervention in the hope of speeding resolution and hence limiting scarring, transmission, and the period of social exclusion. Although many treatments are cited in the literature with destruction of the lesions as their common goal, ${ }^{45}$ a systematic review has revealed little good trial evidence to support them (RMMacS, unpublished observations). We compared the efficacy and cosmetic results of two commonly cited treatments: physical expression by squeezing and chemical ablation with phenol.
\end{abstract}

\section{Participants, methods, and results}

Fourteen (7 male) of 16 patients referred to a paediatric dermatology clinic with at least four molluscum contagiosum lesions entered the trial under parental consent. The average age was 4.6 years (range 7 months to 12 years), and 108 lesions (mean 7.7 (SD 5.0); range 3-16) were studied. Lesions on each patient were photographed and stratified by size, and alternate, similar sized lesions were either treated by physical expression with gloved fingers $(52 / 108)$ or were pierced with a sharpened orange stick impregnated with phenol. Emla cream (Astra, Kings Langley, Hertfordshire), applied one hour previously, provided effective analgesia, and subsequent procedures were well tolerated. One month after treatment, the participants were reviewed by a single observer, and the treated lesions were graded for resolution (unresolved, partial, or complete) and scarring (none, pitting $<1 \mathrm{~mm}$ depth, pitting $\geq 1 \mathrm{~mm}$ depth). Outcome was also dichotomised to contrast (a) complete resolution with no or partial resolution and (b) any degree of scarring with none. Odds ratios, controlling for clustering within participant, were derived from two level, generalised linear mixed models of each binary outcome.

Crude, unadjusted comparison of effect of method of treatment on resolution of 108 molluscum contagiosum lesions and degree of subsequent scarring. Values are numbers (percentage) of lesions by treatment method

\begin{tabular}{|c|c|c|c|c|c|c|}
\hline \multirow[b]{2}{*}{ Treatment } & \multicolumn{3}{|c|}{ Resolution of lesion } & \multicolumn{3}{|c|}{ Degree of scarring or pitting } \\
\hline & Unresolved & Partial & Complete & None & $\begin{array}{c}\text { Pitting } \\
<1 \mathrm{~mm} \text { depth }\end{array}$ & $\begin{array}{c}\text { Pitting } \\
\geqslant 1 \mathrm{~mm} \text { depth }\end{array}$ \\
\hline $\begin{array}{l}\text { Phenol ablation } \\
(n=52)\end{array}$ & $8(15.4)$ & $5(9.6)$ & $39(75.0)$ & $10(19.2)$ & $38(73.1)$ & $4(7.7)$ \\
\hline $\begin{array}{l}\text { Physical expression } \\
(\mathrm{n}=56)\end{array}$ & $4(7.1)$ & $9(16.1)$ & $43(76.8)$ & $35(62.5)$ & $19(33.9)$ & $2(3.6)$ \\
\hline
\end{tabular}

Overall, $76 \%(82 / 108)$ of lesions completely resolved, while only $11 \%(12 / 108)$ exhibited no discernible response. The table presents frequency of resolution and scarring scores by treatment method. No significant differences were observed for either crude resolution scores $(\mathrm{P}=0.33)$ or as a dichotomous outcome when participant effect was controlled for (odds ratio $0.93 ; 95 \%$ confidence interval 0.35 to 2.44 , $\mathrm{P}=0.89)$. However, $63 \%(35 / 56)$ of lesions treated by physical expression showed no scarring, compared with only $19 \%(10 / 52)$ of those treated with phenol $(\mathrm{P}<0.001)$. When participant effect and lesion resolution were controlled for, the unadjusted odds ratio (phenol ablation $v$ physical expression) for the development of any scarring increased from 7.1 (2.7 to $20.0, \mathrm{P}<0.001)$ to 25.5 (6.9 to $93.7, \mathrm{P}<0.001)$.

\section{Comment}

We observed no significant difference between physical expression and phenol ablation in terms of resolution of individual molluscum contagiosum lesions in children; treatment with phenol, however, was significantly more likely to result in scarring. Phenol, however, is consistently cited in the literature as an accepted form of treatment ${ }^{45}$ and is still used by some clinicians to treat molluscum lesions.

Nevertheless, in immunocompetent children, the disease is self limiting, and watchful waiting may be the treatment of choice. Furthermore, the current study did not contrast the two treatment options with watchful waiting, either for efficacy or clinical sequelae. However, it is plausible that early treatment of infection may limit the spread of lesions by autoinoculation. This is an important clinical hypothesis, as early active treatment could thus reduce the duration of infection and the degree of individual scarring and population transmission. Ongoing studies of molluscum infection and treatment may show if this is indeed the case. However, as treatment is usually given for cosmetic reasons, a method that causes considerable scarring is clearly unacceptable.

Contributors: MIW conceived, designed, and supervised the study and reviewed and edited the manuscript. RW assisted in the design, conducted the study, collated the data, and helped to write, review, and edit the manuscript. CJO'C conceived and conducted the analyses of data, interpreted the results, and assisted in the writing and revision of the manuscript. RMMacS wrote the preliminary manuscript and assisted in its revision. MIW will act as guarantor for the paper.

Funding: None.

Competing interests: None declared.

1 Postlethwaite R. Molluscum contagiosum: a review. Arch Environ Health $1970 ; 21: 432-52$

2 Hawley TG. The natural history of molluscum contagiosum in Fijian children.J Hygiene 1970;68:631-2.

3 Field LM, Johnson ML. Ouestions and answers: molluscum contagiosum reaction. JAMA 1980;243:2526.

4 Gottlieb S, Myskowski PL. Molluscum contagiosum. Int J Derm 1994;33: 453-61.

5 Smith EB, Raimer SS. Common viral infections of the skin and their treatment. Med Clin North Am 1982;66:807-17.

(Accepted 7 September 1999) 